\title{
Disease prevalence and shade tree diversity in smallholder cocoa (Theobroma cacao) farms: case of Bundibugyo District, Western Uganda
}

\author{
Blasio Bisereko Bwambale ${ }^{1}$, Godfrey Sseremba ${ }^{1,2}$, Julius Mwine ${ }^{1}$ \\ ${ }^{1}$ Faculty of Agriculture, Uganda Martyrs University, P.O. Box 5498, Kampala, Uganda \\ ${ }^{2}$ National Coffee Research Institute, National Agricultural Research Organization, P.O. Box 185, Mukono, \\ Uganda
}

\begin{abstract}
Cocoa (Theobroma cacao) growing systems in Uganda consists of shade systems with different tree species. Tree shade systems are the pure stand trees in the cocoa plantation which have been attributed to wards reducing on pests and disease incidences, shade provision, boosting fertility, Agro biodiversity, fodder and improving production. The study was aimed at identifying potential shade tree species that can minimize disease threats on cocoa farms. Eighty-two cocoa farmers were reached out of 120 cocoa farmers in Bundibugyo that possessed at least five acres of the plantation in a purposive sampling approach.

Black pod disease was non-significantly associated with presence of shade tree diversities. It was established that incidence of black pod rot disease was non-significantly associated with presence of all shade tree species; association between witch's broom disease incidence with presence of Maesopsis eminii was highly significant $(\chi 2=55.41,(\mathrm{p}<0.05)$; Association between witch's broom and presence of Persea Americana $(\chi 2=9.79), \quad(p<0.05)$, Eucalyptus globulus $(\chi 2=16.71), \quad(p<0.05), \quad$ Markhamia obtusifolia $(\chi 2=3.95),(p<0.001)$, schefflera actinophylla $(\chi 2=4.32),(p<0.001)$, Mangifera indica $(\chi 2=6.46),(p<0.001)$ was significant though these trees were planted in small numbers. Association between frosty rot disease incidence with presence of Maesopsis eminii was highly significant $(\chi 2=25.90),(p<0.05)$. there was a significant association between frosty with presence of Eucalyptus globulus $(\chi 2=6.76),(p<0.05)$; Mangiferaindica $(\chi 2=4.85),(p<0.001)$; though these trees were planted in small numbers. There was a highly significant association between swollen shoot virus with presence of Maesopsis eminii $\left(\chi^{2}=\right.$ $31.55),(p<0.05)$, and Cinnamomum tamala $(\chi 2=19.22),(p<0.05)$. Association between die back disease incidence with presence of Maesopsis eminii was highly significant $(\chi 2=52.73),(p<0.05)$.
\end{abstract}

Keywords: Cocoa farmers; Biodiversity: Shade tree species; Shade systems; and Disease incidence,

\section{Introduction}

The cocoa tree (Theobroma cacao) is highly prized for its beans from which cocoa powder and butter are produced. In the past years, world production tripled to a record of 3.7 million tons of dry cocoa beans in 2008 and generated an estimated US\$7.4 billion of income for millions of smallholder farmers (ICCO, 2008). Cocoa beans are produced globally. In the western world, cocoa is grown in several countries; Brazil $(256,186)$, Ecuador $(128,446)$, Mexico (82,000), Peru (71,175), Dominican Republic68,021, Colombia $(46,739)$, Papua New Guinea $(41,200)$, Venezuela $(31,236)$ in metric tons. In Africa cocoa is produced in the tropical zones around the Equator, where climate conditions are well suited for growing cocoa trees. About 70 percent of the world's cocoa beans come from four West African countries: Ivory Coast, Ghana, Nigeria and Cameroon. The Ivory Coast and Ghana are by far the two largest producers of cocoa, accounting for more than 50 percent of the world's cocoa. In 2016, the Ivory Coast alone produced approximately 1.6 million metric tons of cocoa beans. Uganda produces $(26,000)$ metric tons per year on a total of more than 14,000 hectares had already been planted on smallholdings and is grown in areas of Mukono, Bundibugyo, 
Buikwe, Kibaale, Kasese, Jinja, Kamuli, Iganga, Hoima, Mpigi and Luwero. In Bundibugyo district by 1978, 2 fermenters were established, at Bubomboli, and at the district headquarters. However today according to statistics from the commercial office, more than $85 \%$ of the population is directly dependent on cocoa, and produces about 18,000 metric tons according to District Production Officer Bundibugyo.

Agroforestry is not a new method or a revolutionary way of farming. In fact, this is one of the oldest methods of agricultural production, but it was abandoned for a while because of the intensification of modern agriculture (Nair, 2007). Furthermore, shading trees can be maintained in cocoa based agroforestry systems to reduce pest attacks (Beer et al., 1998) for stabilizing the microclimate (Sporn et al., 2009) for soil protection against rain drops (Dietz et al., 2005). In the scientific literature, there is consensus that cocoabased agroforestry systems with dense and divers shade tree stands do harbor high levels of species richness (Jagoret, 2011). Above all, the aim is not only to sustain the forest in cocoa growing areas but also the increase income of the small-scale cocoa farmers. Production technologies such as canopy and weed management, and disease control were developed to counter the farmers' production problems. These technologies increased cocoa production considerably.

In other studies, tree planting is one of the key objectives to improve food security and well-being of African rural households through agroforestry, this directly via the various types of edible plant, medicinal plants, wood and other non-timber products, and directly through the sustainable provision of environmental service like sequestration and storage of carbon by woody component (cocoa, fruit trees, forest trees) in cocoa-based agroforestry but also disease \& pest control. Different disease incidences vary according to farming systems. Here, the attention of cocoa diseases evaluation in a cocoa tree shade system is drawn to come out with a better and sustainable system for cocoa production while protecting the environment and conserving biodiversity.

There is quite a number of diseases which are common in cocoa production in Uganda and specifically Bundibugyo. Phytophthora Pod Rot also known as Black pod, is caused by the fungus Phytophthora spp. causes global yield loss of $20-30 \%$ and tree deaths of $10 \%$ annually and causing significant losses in favorable environments. Frosty Pod Rot is caused by the basidiomycete moniliophthora roreri, first reports of the disease date back to the end of the $19^{\text {th }}$ century, where its aggressive effects cause devastation in cocoa plantations. Witch's broom -Moniliophthora perniciosa is a fungus responsible for Witch's broom disease. The fungus attacks only growing tissues that is, shoots, flowers and pods causing cocoa trees to produce branches with no fruit and ineffective leaves. The pods show distortion and present green patches that give the appearance of uneven ripening. Dieback disease, it caused heavy losses of mature trees and seedlings planted near older cocoa. it has been a major problem in the large commercial plantations in Uganda but also in other countries like West Malaysia and Sabah

Shade trees have been observed as part and partial in cocoa production to boost production, disease control, biodiversity and environmental conservation among others. Specific trees have been thought of; palm trees these have often been grown with cocoa plantations in countries like Ghana and Uganda. Maesopsis eminii, coconut (Cocos nucifera), neem (Azadirachta indica), mangoes (Mangifera indica), tamarind (Tamarindus indica), Jack fruit (Artocarpus heterophyllus) and avocado (Persea Americana) trees grown as multipurpose trees for shade as well fruits for home consumption. However, the exotic avocado has been known host for cocoa diseases in Southeast Asia.

There has been little research on this cocoa shade trees regarding disease association. Therefore, the main objective of this study was to identify shade tree species possessing potential for reducing disease constraints in cocoa farms. Specifically, we aimed at determining the influence of: (i) shade tree species diversity on incidence of diseases in cocoa; and (ii) shade tree species on the incidence of diseases in cocoa.

\section{Materials and methods}

\subsection{Study design}

The study used a cross sectional research design to collect data one point at a time (Sekeran, 2003). The study triangulated quantitative and qualitative methods, the quantitative approach was used to quantify findings using measures of central tendency, correlation and regression techniques while as qualitative approach was used to gain explanations for any phenomenon. 
The study was looked at how specific objectives were answered; this was done through applying different methods of data collection (photographing, observations and questionnaires). Each method was employed where applicable depending on which variable in question.

\subsection{Study area}

The study took place in Bundibugyo district, and 4 sub-counties were selected where respondents considered for qualitative \& quantitative data were selected using judgmental sampling. Ngamba S/C located in the southern part of the district, Busaru S/C central part of the district, Buganikere T/C located in the central region of the district, Mirambi S/C located in eastern side of the district, which is 1 from Bughendera County located in the southern part of Bundibugyo and 3 from Bwamba County located in the northern side.

\subsection{Target population, sampling frame and sample size}

Uganda is estimated to have 20,000 hectares of land for cocoa growing, although there is no clear data on the number of farmers growing cocoa (https://www.aboutuganda.com/uganda/agriculture/cocoa-growing-inuganda). Bundibugyo community has a predominantly agricultural population estimated at about 60,000, with a density of 150 inhabitants per square kilometre. For Bundibugyo, data on the exact population number has not been available; it has been estimated at 30,000 households in the district and about 42,000 farmers are involved in cocoa farming according to Production Officer Bundibugyo. However, the study area had 7,190 cocoa farmers and current farmers register indicated 120 farmers with five acres and above in the study area. Eighty-two (82) respondents (Table 1) were reached out of 120 cocoa farmers who cultivate on less than five acres of land (Word Bank, 2016) reflecting a response rate of 68.3\%. The respondents were selected from four sub counties namely Mirambi, Ngamba, Buganikere and Busaru

Table1 Number of cocoa farmers in the selected sub counties

\begin{tabular}{|c|c|c|c|}
\hline $\begin{array}{c}\text { Sub county } \\
\text { name }\end{array}$ & $\begin{array}{c}\text { No. of cocoa } \\
\text { farmers }\end{array}$ & $\begin{array}{c}\text { No. selected } \\
\text { respondents }\end{array}$ & Actual respondents \\
\hline Mirambi & 2200 & 36 & 21 \\
\hline Ngamba & 1450 & 24 & 19 \\
\hline Busaru & 1890 & 31 & 21 \\
\hline Buganikere & 1650 & 27 & 21 \\
\hline Total & $\mathbf{7 , 1 9 0}$ & $\mathbf{1 1 8}$ & $\mathbf{8 2}$ \\
\hline
\end{tabular}

\subsection{Data collection}

\subsubsection{Influence of shade tree diversity on disease incidence}

Data was collected at household level, considering individual farmers and basically this was primary data derived direct from the cocoa farmers to whom questioners were administered. various methods were employed during data collection not limited to, questionnaires, observations and photograph.

Questionnaires, these were developed and administered at farmer level. This tool was able to capture the diseases types and the rate of attack against shade diversity. After identifying the sample size, every farmer was subjected to answer the questionnaire. Questionnaire was used because of its ability to collect vast data and gave various options to selected respondents to make their selection (Amin, 2005). Questionnaires were aided by photos of specific diseases to enable respondents give valid information as indicated in the appendix. The sample questions were but not limited to; Do cocoa diseases attack your farm, which type of cocoa diseases attack your farm, how many types of trees do you have on your cocoa farm?

Observations; specifically, the study also required observing specific cases and through this, visual data were recorded which helped in enriching this thesis. This focused on areas of; signs and symptoms of cocoa diseases, extent of damages caused by cocoa diseases.

Photography; pictures were taken in form of data that was collected and compiled to enrich the dissertation.

\subsubsection{Effect of shade tree species on disease incidence}


Data was collected at household level, considering individual farmers and basically this was primary data derived direct from the cocoa farmers.

Questionnaires were developed and administered at farmer level - respondents for this research. This tool captured the diseases types and the specific tree species. The sample questions were; which type of trees species do you grow on your farm, which type of cocoa diseases attack your farm, how often do cocoa diseases attack your farm?

Observations were used to observing specific disease symptoms, visual data were recorded which helped in enriching this thesis. Tree species on farm were observed, signs and symptoms of cocoa diseases.

\subsection{Data analysis}

\subsubsection{Influence of shade tree diversity on disease incidence}

The questionnaire was pre-tested using a selected group of respondents from the target population to ensure reliability and validity of the questions and responses. All data were coded and analysis was carried using descriptive statistics where statistical package SPSS was used to obtain the chai squared test values of perceptions obtained from respondents. Data was presented in Bar graphs to help simplify analysis and analysis was mainly done using descriptive analysis where issues of association and significance of | responses were compared.

\subsubsection{Effect of shade tree species on disease incidence}

The questionnaire was pre-tested using a selected group of respondents from the target population to ensure reliability and validity of the questions and responses. All data were coded and analysis was carried using descriptive statistics where statistical package SPSS was used to obtain chai squared test values of perceptions from respondents._Data was presented in tables to help simplify analysis and analysis was mainly done using descriptive analysis to obtain chai squared values and the level of significance was at $95 \%$

\section{Results \\ 3.1 Influence of shade tree diversity on disease incidence \\ Black pod rot disease}

The association between shade diversity and disease incidence was non-significant (Fig 1). Regardless of the different shade diversity systems recorded from several respondents, the disease incidence was reported by all respondents. Therefore, this means that with or without the presence of shade diversity, black pod rot disease incidence would still be present on these farms.

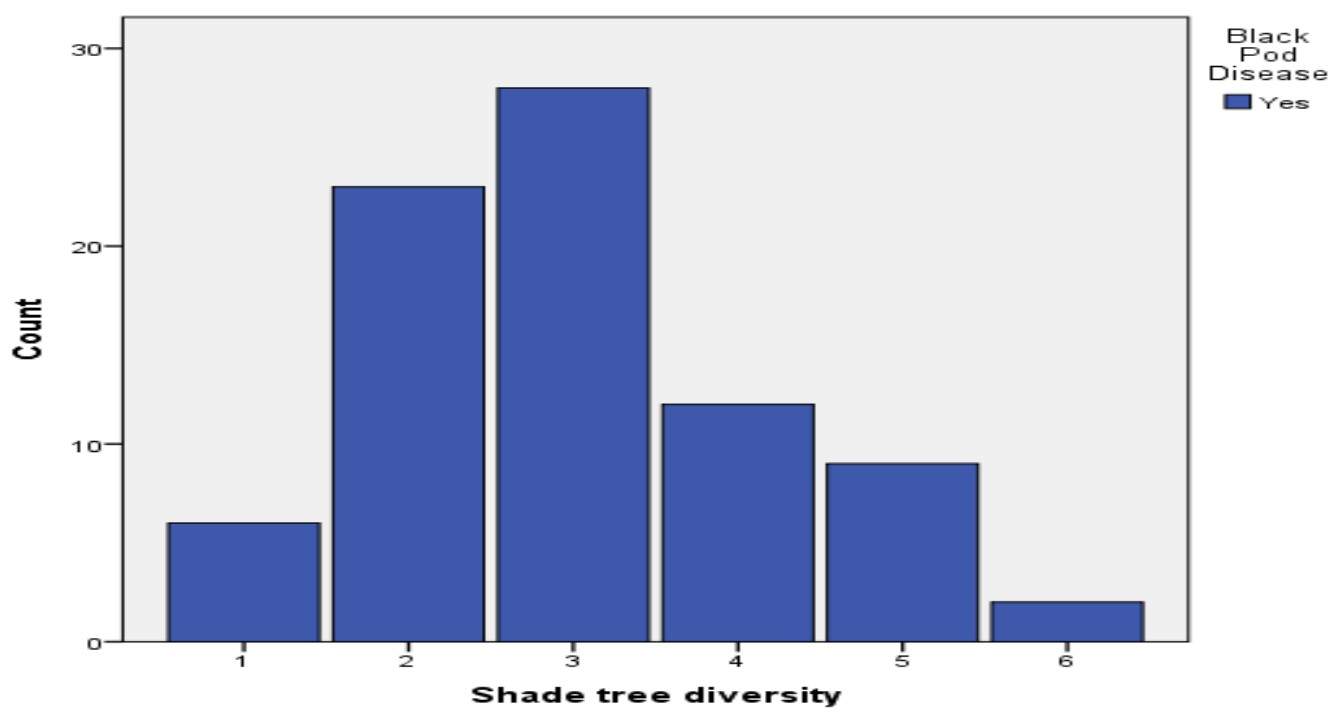

Figure 1: Black Pod rot disease in association with shade tree diversity 
Shade diversity 1 had 8 farmers, 3 reported disease incidences on their farms and 5 did not have the disease. Shade diversity 2 had 27 farmers, 14 farmers reported disease incidence while 13 farmers did not report the disease on their farms. Shade diversity 3 had 35 farmers, 15 reported the disease presence and 20 did not have disease. Shade diversity 4 had 14 farmers, 6 reported disease presence and 8 did not report the disease on their farms. Shade diversity 5 had 9 farmers and all reported disease incidence. Shade system 6 had 2 farmers and both reported disease incidence. (Fig 2)

There is a significant association between the shade diversity 1,2,3\&4 and witch's broom disease. At diversity $3 \& 4$ respectively, the disease incidence cases were lower than the normal cases. association between the disease incidence and shade diversity 5\&6 was insignificant (Fig 2) Only diseased cases were reported by respondents.

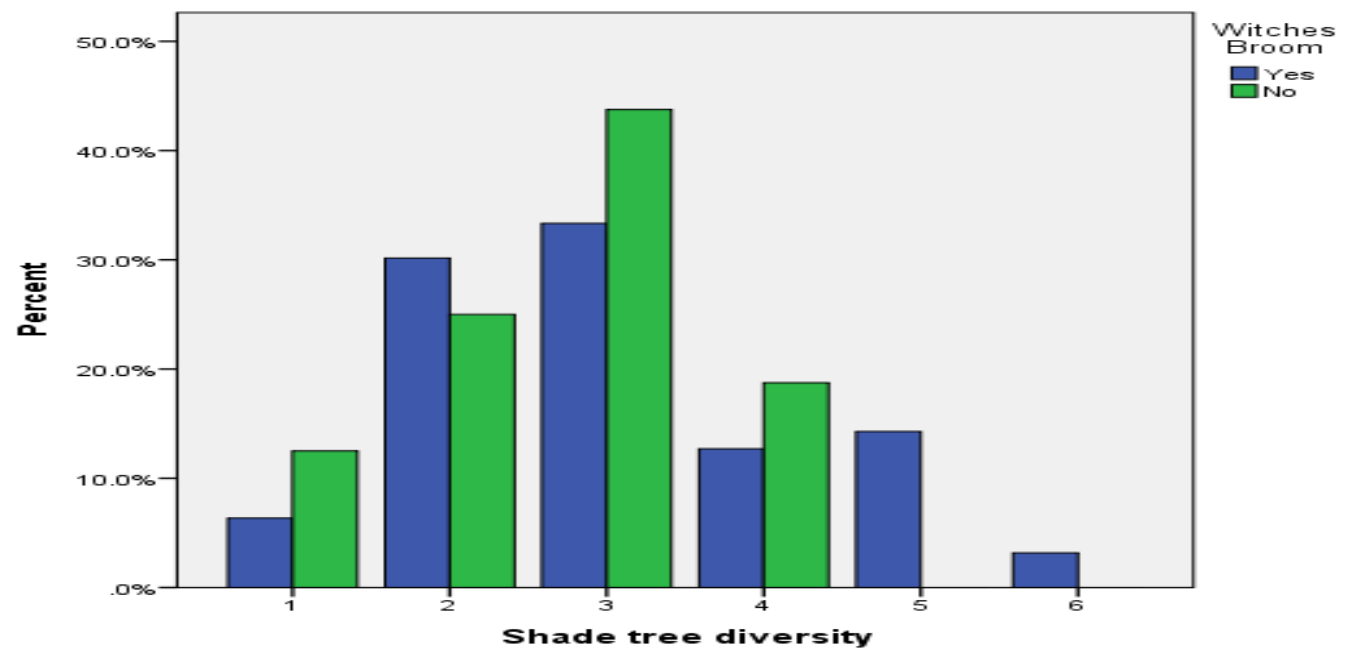

Figure 2: Witch's Broom disease in association with shade tree diversity

\section{Frost pod rot disease}

The analysis of the frost pod rot disease incidence in (Fig 3), followed several tends; Shade diversity 1-3 there are influences noticed though not significant. Shade diversity 4-6 had completely no influence on disease incidence.

The disease incidence case across all shade diversity suppressed by far the normal case (shade diversity $1,2,3)$ and at shade diversity 4,5,6, disease incidence was at the peak

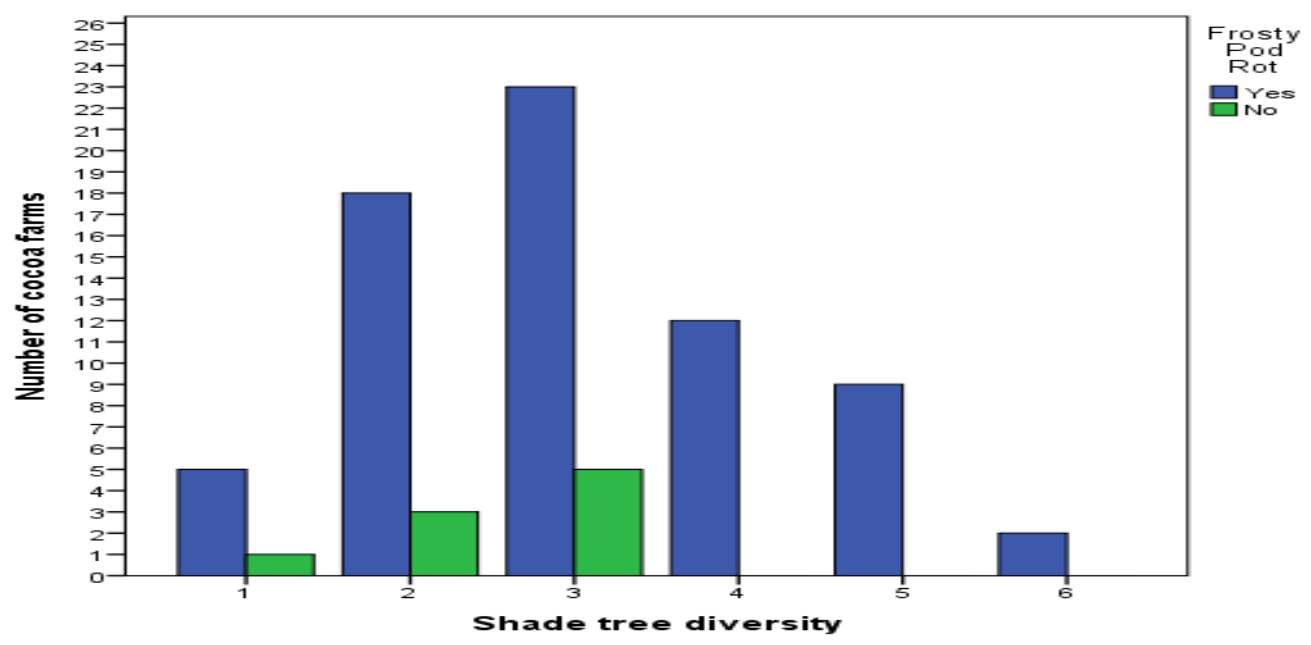

Figure 3:Frost pod rot disease incidence with shade tree diversity

\section{Cocoa Swollen shoot virus}

The analysis of cocoa swollen shoot virus (Fig 4); there is quite significant levels at each shade diversity. The disease prevalence at shade diversity $1,2,3,4 \& 5$ is negatively influenced. This means these shade 
systems limited in some way the occurrence of the disease. Unlike shade diversity 6 which had no influence on the disease occurrence. Disease prevalence was reported across all shade tree diversities. However, shade diversity $1,2,3,4 \& 5$ had an influence on the on the disease prevalence as opposed to shade diversity 6

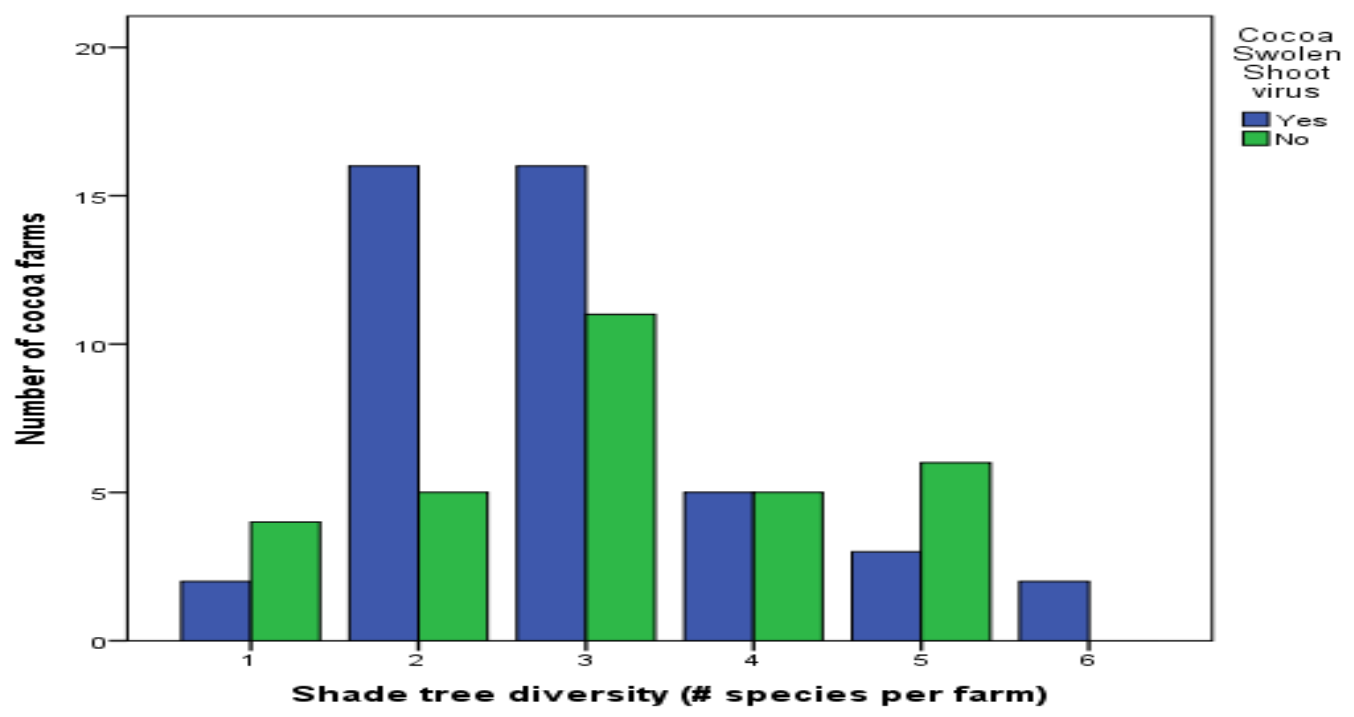

Figure 4:Cocoa Swollen shoot virus prevalence in shade tree diversity

\section{Cocoa Dieback Disease}

Although there are bits of un diseased cases at shade diversity, 1.2.3\&4, the association between cocoa die back disease and shade diversity are insignificant. The diseased cases were far surpassing un diseased cases. (Fig 5). And at shade diversity 5\&6, there was no influence in limiting disease incidence

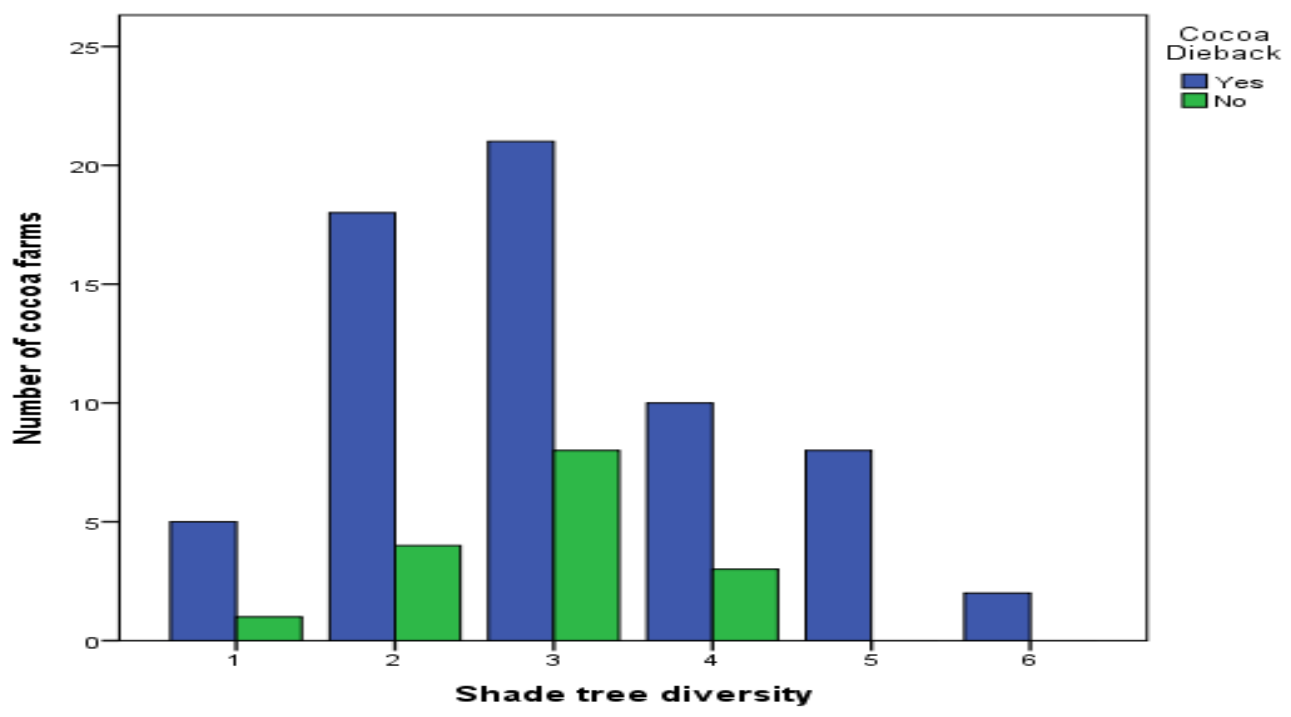

Figure 5: Cocoa Dieback in association with shade tree diversity

\subsection{Effect of shade tree species on disease incidence}

\section{Black pod rot disease with shade tree species}

Black pod rot disease incidence was present in all tree shade species as represented by $100 \%$ (all farms) (Table 2). Farmers asserted that the diseases incidence occurred on all farms. However, farmers with Maesopsis eminii registered the highest number of 58 disease incidence cases, followed by Persea Americana 29, Artocarpus heterophyllus 27, Cinnamomum tamala 23, Eucalyptus globulus 23, Mangifera indica14, Spathodea campanulata 9

Table 2: Analysis of black pod rot disease with shade tree species in Bundibugyo District

\begin{tabular}{|l|l|l|l|l|l|}
\hline Shade tree species & Disease presence & Total & Shade tree species & Disease presence & Total \\
\hline
\end{tabular}




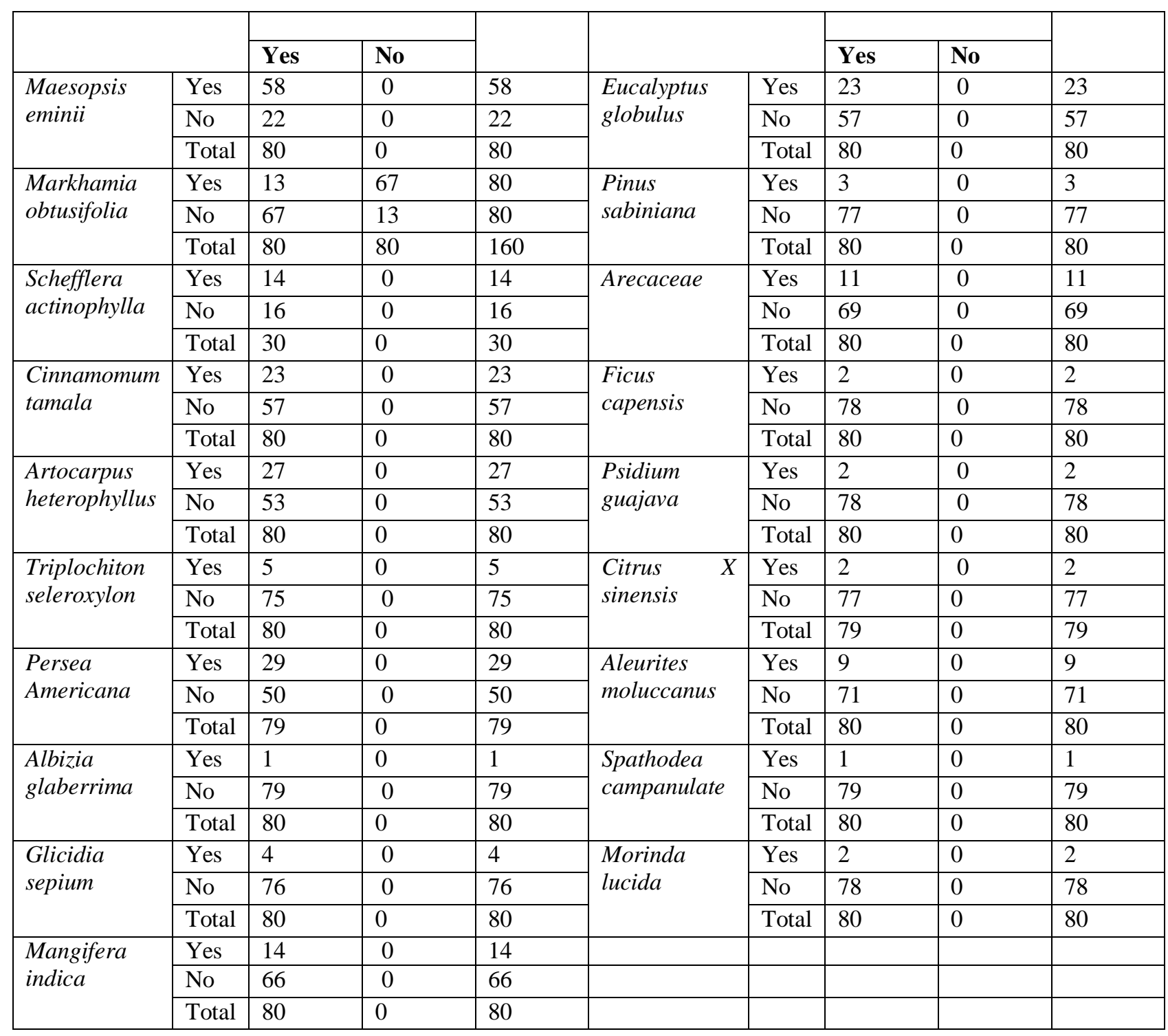

\section{Association of witch's Brom disease in shade tree species}

The association between witch's broom and presence of Maesopsis eminii was highly significant $(\mathrm{x} 2=55.41),(p<0.05),($ Table 3). Association between witch's broom and presence of Persea Americana $(\mathrm{x} 2=9.79), \quad(p<0.05)$, Eucalyptus globulus $(\mathrm{x} 2=16.71), \quad(p<0.05)$, Markhamia obtusifolia $(\mathrm{x} 2=3.95)$, $(p<0.001)$, schefflera actinophylla $(\mathrm{x} 2=4.32),(p<0.001)$, Mangifera indica $(\mathrm{x} 2=6.46),(p<0.001)$ was significant for disease incidence though these trees were planted in small numbers as indicated in the table below. There was a non-significant association between witch's broom incidence with presence of Artocarpus heterophyllus, Triplochiton seleroxylom, Albizia glaberrima, Triplochiton seleroxylom, Pinus sabiniana, Arecaceae, Psidium guajava, Citrus X sinensis, Aleurites moluccanus, Spathodea campanulate, Morinda lucida.

Table 3: Association of Witch's Broom in shade tree species

\begin{tabular}{|c|c|c|c|c|c|c|c|c|c|c|c|}
\hline \multicolumn{2}{|c|}{ Shade tree species } & \multicolumn{2}{|c|}{$\begin{array}{l}\text { Disease } \\
\text { presence }\end{array}$} & \multirow[t]{2}{*}{ Total } & \multirow[t]{2}{*}{$\begin{array}{l}\text { Chi- } \\
\text { squared }\end{array}$} & \multirow{2}{*}{\multicolumn{2}{|c|}{ Shade tree species }} & \multicolumn{2}{|c|}{$\begin{array}{l}\text { Disease } \\
\text { presence }\end{array}$} & \multirow[t]{2}{*}{ Total } & \multirow[t]{2}{*}{$\begin{array}{l}\text { Chi- } \\
\text { squared }\end{array}$} \\
\hline & & \multirow{2}{*}{$\begin{array}{l}\text { Yes } \\
58\end{array}$} & \multirow{2}{*}{$\begin{array}{l}\text { No } \\
0\end{array}$} & & & & & \multirow{2}{*}{$\begin{array}{l}\text { Yes } \\
11\end{array}$} & \multirow{2}{*}{$\begin{array}{l}\text { No } \\
1\end{array}$} & & \\
\hline \multirow{3}{*}{$\begin{array}{l}\text { Maesopsis } \\
\text { eminii }\end{array}$} & Yes & & & 58 & \multirow[t]{3}{*}{$55.41 * * *$} & \multirow{3}{*}{$\begin{array}{l}\text { Eucalyptus } \\
\text { globulus }\end{array}$} & Yes & & & 22 & \multirow[t]{3}{*}{16.71 *** } \\
\hline & No & 5 & 16 & 21 & & & No & 52 & 5 & 57 & \\
\hline & Total & 63 & 16 & 79 & & & Total & 63 & 16 & 79 & \\
\hline \multirow{3}{*}{$\begin{array}{l}\text { Markhamia } \\
\text { obtusifolia }\end{array}$} & Yes & 13 & 0 & 13 & \multirow[t]{3}{*}{$3.95 * *$} & \multirow{3}{*}{$\begin{array}{l}\text { Pinus } \\
\text { sabiniana }\end{array}$} & Yes & 3 & 0 & 3 & \multirow[t]{3}{*}{0.79} \\
\hline & No & 50 & 16 & 66 & & & No & 60 & 16 & 76 & \\
\hline & Total & 63 & 16 & 79 & & & Total & 63 & 16 & 79 & \\
\hline Schefflera & Yes & 14 & 0 & 14 & $4.32 *$ & Arecaceae & Yes & 6 & 4 & 10 & 2.76 \\
\hline
\end{tabular}




\begin{tabular}{|c|c|c|c|c|c|c|c|c|c|c|c|}
\hline \multirow[t]{2}{*}{ actinophylla } & No & 49 & 16 & 65 & & & No & 57 & 12 & 69 & \\
\hline & Total & 63 & 16 & 79 & & & Total & 63 & 16 & 79 & \\
\hline \multirow{3}{*}{$\begin{array}{l}\text { Artocarpus } \\
\text { heterophyllus }\end{array}$} & Yes & 18 & 8 & 26 & \multirow[t]{3}{*}{2.65} & \multirow{3}{*}{$\begin{array}{l}\text { Ficus } \\
\text { capensis }\end{array}$} & Yes & 2 & 0 & 2 & \multirow[t]{3}{*}{0.52} \\
\hline & No & 45 & 8 & 53 & & & No & 61 & 16 & 77 & \\
\hline & Total & 63 & 16 & 79 & & & Total & 63 & 16 & 79 & \\
\hline \multirow{3}{*}{$\begin{array}{l}\text { Triplochiton } \\
\text { seleroxylon }\end{array}$} & Yes & 4 & 1 & 5 & \multirow[t]{3}{*}{0} & \multirow{3}{*}{$\begin{array}{l}\text { Psidium } \\
\text { guajava }\end{array}$} & Yes & 2 & 0 & 2 & \multirow[t]{3}{*}{0.52} \\
\hline & No & 59 & 15 & 74 & & & No & 61 & 16 & 77 & \\
\hline & Total & 63 & 16 & 79 & & & Total & 63 & 16 & 79 & \\
\hline \multirow{3}{*}{$\begin{array}{l}\text { Persea } \\
\text { Americana }\end{array}$} & Yes & 17 & 11 & 28 & \multirow[t]{3}{*}{$9.79 * * *$} & \multirow{3}{*}{$\begin{array}{ll}\text { Citrus } & X \\
\text { sinensis } & \end{array}$} & Yes & 1 & 1 & 2 & \multirow[t]{3}{*}{5.19} \\
\hline & No & 45 & 5 & 50 & & & No & 62 & 14 & 76 & \\
\hline & Total & 62 & 16 & 78 & & & Total & 63 & 15 & 78 & \\
\hline \multirow{3}{*}{$\begin{array}{l}\text { Albizia } \\
\text { glaberrima }\end{array}$} & Yes & 1 & 0 & 1 & \multirow[t]{3}{*}{0.26} & \multirow{3}{*}{$\begin{array}{l}\text { Aleurites } \\
\text { moluccanus }\end{array}$} & Yes & 9 & 0 & 9 & \multirow[t]{3}{*}{2.58} \\
\hline & No & 62 & 16 & 78 & & & No & 54 & 16 & 70 & \\
\hline & Total & 63 & 16 & 79 & & & Total & 63 & 16 & 79 & \\
\hline \multirow{3}{*}{$\begin{array}{l}\text { Glicidia } \\
\text { sepium }\end{array}$} & Yes & 5 & 0 & 5 & \multirow[t]{3}{*}{1.36} & \multirow{3}{*}{$\begin{array}{l}\text { Spathodea } \\
\text { campanulata }\end{array}$} & Yes & 1 & 0 & 1 & \multirow[t]{3}{*}{0.26} \\
\hline & No & 58 & 16 & 74 & & & No & 62 & 16 & 78 & \\
\hline & Total & 63 & 16 & 79 & & & Total & 63 & 16 & 79 & \\
\hline \multirow{3}{*}{$\begin{array}{l}\text { Mangifera } \\
\text { indica }\end{array}$} & Yes & 5 & 6 & 11 & \multirow[t]{3}{*}{$6.46^{*}$} & \multirow{3}{*}{$\begin{array}{l}\text { Morinda } \\
\text { lucida }\end{array}$} & Yes & 2 & 0 & 2 & \multirow[t]{3}{*}{0.52} \\
\hline & No & 56 & 10 & 66 & & & No & 61 & 16 & 77 & \\
\hline & Total & 61 & 16 & 77 & & & Total & 63 & 16 & 79 & \\
\hline
\end{tabular}

$*, * *$ and $* * *$ stand for significance at 5,1 and $0.1 \%$ error margin, respectively

\section{Prevalence of frosty pod rot disease with shade tree species}

Prevalence of frost pod rot disease was highly associated with presence of Maesopsis eminii $\left(\mathrm{X}^{2}=25.90\right)$, $(p<0.05)$; Eucalyptus globulus $\left(\mathrm{X}^{2}=6.76\right),(p<0.05)$; planted $6 / 44$ farms, Mangifera indica $\left(\mathrm{X}^{2}=4.85\right)$, $(p<0.001)$; were not responsible for disease incidence since they were planted in small numbers. Presence of Schefflera actinophylla, Artocarpus heterophyllus, Triplochiton seleroxylon, Persea Americana, Arecaceae tree species, Ficus capensis, Psidium guajava, Glicidia sepium, Citrus X sinensis, Aleurites moluccanus, Spathodea campanulate, Morinda lucida, Pinus sabiniana had non-significant association for disease incidence.

Table 4: Prevalence of Frost Pod Rot Disease with shade tree species

\begin{tabular}{|c|c|c|c|c|c|c|c|c|c|c|c|}
\hline \multirow{2}{*}{\multicolumn{2}{|c|}{ Shade tree species }} & \multicolumn{2}{|c|}{$\begin{array}{l}\text { Disease } \\
\text { presence }\end{array}$} & \multirow[t]{2}{*}{ Total } & \multirow[t]{2}{*}{$\begin{array}{l}\text { Chi- } \\
\text { squared }\end{array}$} & \multirow{2}{*}{\multicolumn{2}{|c|}{ Shade tree species }} & \multicolumn{2}{|c|}{$\begin{array}{l}\text { Disease } \\
\text { presence }\end{array}$} & \multirow[t]{2}{*}{ Total } & \multirow[t]{2}{*}{$\begin{array}{l}\text { Chi- } \\
\text { squared }\end{array}$} \\
\hline & & \multirow{2}{*}{$\begin{array}{l}\text { Yes } \\
56\end{array}$} & \multirow{2}{*}{$\begin{array}{l}\text { No } \\
0\end{array}$} & & & & & \multirow{2}{*}{$\begin{array}{l}\text { Yes } \\
17\end{array}$} & No & & \\
\hline \multirow{3}{*}{$\begin{array}{l}\text { Maesopsis } \\
\text { eminii }\end{array}$} & Yes & & & 56 & \multirow[t]{3}{*}{$25.90 * * *$} & \multirow{3}{*}{$\begin{array}{l}\text { Eucalyptus } \\
\text { globulus }\end{array}$} & Yes & & 6 & 23 & \multirow[t]{3}{*}{$6.76 * * *$} \\
\hline & No & 13 & 9 & 22 & & & No & 52 & 3 & 55 & \\
\hline & Total & 69 & 9 & 78 & & & Total & 69 & 9 & 78 & \\
\hline \multirow{3}{*}{$\begin{array}{l}\text { Markhamia } \\
\text { obtusifolia }\end{array}$} & Yes & 8 & 4 & 12 & \multirow[t]{3}{*}{2.04} & \multirow{3}{*}{$\begin{array}{l}\text { Pinus } \\
\text { sabiniana }\end{array}$} & Yes & 3 & 0 & 3 & \multirow[t]{3}{*}{0.41} \\
\hline & No & 36 & 27 & 63 & & & No & 66 & 9 & 75 & \\
\hline & Total & 44 & 31 & 75 & & & Total & 69 & 9 & 78 & \\
\hline \multirow{3}{*}{$\begin{array}{l}\text { Schefflera } \\
\text { actinophylla }\end{array}$} & Yes & 14 & 0 & 14 & \multirow[t]{3}{*}{2.23} & \multirow[t]{3}{*}{ Arecaceae } & Yes & 10 & 1 & 11 & \multirow[t]{3}{*}{0.08} \\
\hline & No & 55 & 9 & 64 & & & No & 59 & 8 & 67 & \\
\hline & Total & 69 & 9 & 78 & & & Total & 69 & 9 & 78 & \\
\hline \multirow{3}{*}{$\begin{array}{l}\text { Artocarpus } \\
\text { heterophyllus }\end{array}$} & Yes & 24 & 3 & 27 & \multirow[t]{3}{*}{0.01} & \multirow{3}{*}{$\begin{array}{l}\text { Ficus } \\
\text { capensis }\end{array}$} & Yes & 2 & 0 & 2 & \multirow[t]{3}{*}{0.27} \\
\hline & No & 45 & 6 & 51 & & & No & 67 & 9 & 76 & \\
\hline & Total & 69 & 9 & 78 & & & Total & 69 & 9 & 78 & \\
\hline \multirow{3}{*}{$\begin{array}{l}\text { Triplochiton } \\
\text { seleroxylon }\end{array}$} & Yes & 5 & 0 & 5 & \multirow[t]{3}{*}{0.71} & \multirow{3}{*}{$\begin{array}{l}\text { Psidium } \\
\text { guajava }\end{array}$} & Yes & 2 & 0 & 2 & \multirow[t]{3}{*}{0.27} \\
\hline & No & 65 & 9 & 74 & & & No & 67 & 9 & 76 & \\
\hline & Total & 70 & 9 & 79 & & & Total & 69 & 9 & 78 & \\
\hline \multirow{3}{*}{$\begin{array}{l}\text { Persea } \\
\text { Americana }\end{array}$} & Yes & 23 & 6 & 29 & 3.83 & Citrus & Yes & 1 & 1 & 2 & 3.09 \\
\hline & No & 45 & 3 & 48 & & sinensis & No & 67 & 8 & 75 & \\
\hline & Total & 68 & 9 & 77 & & & Total & 68 & 9 & 77 & \\
\hline Albizia & Yes & 1 & 0 & 1 & 0.13 & Aleurites & Yes & 9 & 0 & 9 & 1.32 \\
\hline
\end{tabular}




\begin{tabular}{|c|c|c|c|c|c|c|c|c|c|c|c|}
\hline \multirow[t]{2}{*}{ glaberrima } & No & 68 & 9 & 77 & & \multirow[t]{2}{*}{ moluccanus } & No & 60 & 9 & 69 & \\
\hline & Total & 69 & 9 & 78 & & & Total & 69 & 9 & 78 & \\
\hline \multirow{3}{*}{$\begin{array}{l}\text { Glicidia } \\
\text { sepium }\end{array}$} & Yes & 4 & 0 & 4 & \multirow[t]{3}{*}{0.55} & \multirow{3}{*}{$\begin{array}{l}\text { Spathodea } \\
\text { campanulata }\end{array}$} & Yes & 1 & 0 & 1 & \multirow[t]{3}{*}{0.13} \\
\hline & No & 65 & 9 & 74 & & & No & 68 & 9 & 77 & \\
\hline & Total & 69 & 9 & 78 & & & Total & 69 & 9 & 78 & \\
\hline \multirow{3}{*}{$\begin{array}{l}\text { Mangifera } \\
\text { indica }\end{array}$} & Yes & 10 & 4 & 14 & \multirow[t]{3}{*}{$4.85 *$} & \multirow{3}{*}{$\begin{array}{l}\text { Morinda } \\
\text { lucida }\end{array}$} & Yes & 2 & 0 & 2 & \multirow[t]{3}{*}{0.27} \\
\hline & No & 59 & 5 & 64 & & & No & 67 & 9 & 76 & \\
\hline & Total & 69 & 9 & 78 & & & Total & 69 & 9 & 78 & \\
\hline
\end{tabular}

$*, * *$ and $* * *$ stand for significance at 5,1 and $0.1 \%$ error margin, respectively

\section{Association of cocoa swollen shoot virus disease with shade tree species}

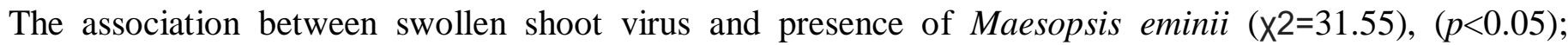
Cinnamomum tamala $\left(x_{2}=19.22\right),(p<0.05)$; was highly significant. (Table 5). Association between swollen shoot virus and Eucalyptus globulus $(\mathrm{X} 2=14.10),(p<0.05)$; Persea Americana $(\mathrm{X} 2=11.17),(p<0.05)$; Aleurites moluccanus $(\mathrm{X} 2=7.21),(p<0.05)$; was significant though these tree species were planted in small numbers (Table 5). Markhamia obtusifolia, Schefflera actinophylla, Artocarpus heterophyllus, Triplochiton seleroxylem, Albizia glaberrima, Glicidia sepium, Mangifera indica, Pinus sabiniana, Arecaceae, Ficus capensis, Psidium guajava, Citrus X sinensis, Spathodea campanulate, Morinda lucida were nonsignificance for disease incidence.

Table 5: Prevalence of swollen Shoot Virus Disease in tree species

\begin{tabular}{|c|c|c|c|c|c|c|c|c|c|c|c|}
\hline \multirow{2}{*}{\multicolumn{2}{|c|}{ Shade tree species }} & \multicolumn{2}{|c|}{$\begin{array}{l}\text { Disease } \\
\text { presence }\end{array}$} & \multirow[t]{2}{*}{ Total } & \multirow[t]{2}{*}{$\begin{array}{l}\text { Chi- } \\
\text { squared }\end{array}$} & \multirow{2}{*}{\multicolumn{2}{|c|}{ Shade tree species }} & \multicolumn{2}{|c|}{$\begin{array}{l}\text { Disease } \\
\text { presence }\end{array}$} & \multirow[t]{2}{*}{ Total } & \multirow[t]{2}{*}{$\begin{array}{l}\text { Chi- } \\
\text { squared }\end{array}$} \\
\hline & & \multirow{2}{*}{$\begin{array}{l}\text { Yes } \\
42\end{array}$} & \multirow{2}{*}{$\begin{array}{l}\text { No } \\
11\end{array}$} & & & & & \multirow{2}{*}{\begin{tabular}{|l|} 
Yes \\
6
\end{tabular}} & No & & \\
\hline \multirow{3}{*}{$\begin{array}{l}\text { Maesopsis } \\
\text { eminii }\end{array}$} & Yes & & & 53 & \multirow[t]{3}{*}{$31.55 * * *$} & \multirow{3}{*}{$\begin{array}{l}\text { Eucalyptus } \\
\text { globulus }\end{array}$} & Yes & & 17 & 23 & \multirow[t]{3}{*}{$14.52 * * *$} \\
\hline & No & 2 & 20 & 22 & & & No & 38 & 14 & 52 & \\
\hline & Total & 44 & 31 & 75 & & & Total & 44 & 31 & 23 & \\
\hline \multirow{3}{*}{$\begin{array}{l}\text { Markhamia } \\
\text { obtusifolia }\end{array}$} & Yes & 8 & 4 & 12 & \multirow[t]{3}{*}{0.38} & \multirow{3}{*}{\begin{tabular}{|l|} 
Pinus \\
sabiniana
\end{tabular}} & Yes & 4 & 1 & 5 & \multirow[t]{3}{*}{0.08} \\
\hline & No & 36 & 7 & 43 & & & No & 42 & 30 & 72 & \\
\hline & Total & 44 & 11 & 55 & & & Total & 46 & 31 & 77 & \\
\hline \multirow{3}{*}{$\begin{array}{l}\text { Schefflera } \\
\text { actinophylla }\end{array}$} & Yes & 5 & 7 & 12 & \multirow[t]{3}{*}{1.7} & \multirow[t]{3}{*}{ Arecaceae } & Yes & 4 & 7 & 11 & \multirow[t]{3}{*}{2.64} \\
\hline & No & 39 & 24 & 63 & & & No & 40 & 24 & 64 & \\
\hline & Total & 44 & 31 & 75 & & & Total & 44 & 31 & 75 & \\
\hline \multirow{3}{*}{$\begin{array}{l}\text { Cinnamomum } \\
\text { tamala }\end{array}$} & Yes & 20 & 0 & 20 & \multirow[t]{3}{*}{$19.22 * * *$} & \multirow{3}{*}{\begin{tabular}{|l|} 
Ficus \\
capensis
\end{tabular}} & Yes & 1 & 1 & 2 & \multirow[t]{3}{*}{0.06} \\
\hline & No & 24 & 31 & 55 & & & No & 43 & 30 & 73 & \\
\hline & Total & 44 & 31 & 75 & & & Total & 44 & 31 & 75 & \\
\hline Artocarpus & Yes & 13 & 14 & 27 & 1.93 & Psidium & Yes & 1 & 1 & 2 & 0.06 \\
\hline heterophyllus & No & 31 & 17 & 48 & & guajava & No & 43 & 30 & 73 & \\
\hline & Total & 44 & 31 & 75 & & & Total & 44 & 31 & 75 & \\
\hline Triplochiton & Yes & 4 & 3 & 7 & 0.8 & Citrus & Yes & 1 & 1 & 2 & 1.51 \\
\hline seleroxylon & No & 42 & 28 & 70 & & sinensis & No & 43 & 29 & 72 & \\
\hline & Total & 46 & 31 & 77 & & & Total & 44 & 30 & 74 & \\
\hline Persea & Yes & 9 & 18 & 27 & $11.47 * * *$ & \begin{tabular}{|l} 
Aleurites \\
\end{tabular} & Yes & 9 & 0 & 9 & $7.21 * * *$ \\
\hline Americana & No & 34 & 13 & 47 & & moluccanus & No & 35 & 31 & 66 & \\
\hline & Total & 43 & 31 & 74 & & & Total & 44 & 31 & 75 & \\
\hline Albizia & Yes & 0 & 1 & 1 & 1.44 & Spathodea & Yes & 1 & 0 & 1 & 0.71 \\
\hline glaberrima & No & 44 & 30 & 74 & & campanulata & No & 43 & 31 & 74 & \\
\hline & Total & 44 & 31 & 75 & & & Total & 44 & 31 & 75 & \\
\hline Glicidia & Yes & 3 & 0 & 3 & 2.2 & Morinda & Yes & 2 & 0 & 2 & 1.45 \\
\hline sepium & No & 42 & 31 & 73 & & lucida & No & 42 & 31 & 73 & \\
\hline & Total & 45 & 31 & 76 & & & Total & 44 & 31 & 75 & \\
\hline Mangifera & Yes & 6 & 7 & 13 & 1.02 & & & & & & \\
\hline
\end{tabular}




\begin{tabular}{|l|l|l|l|l|l|l|l|l|l|l|l|}
\hline \multirow{2}{*}{ indica } & No & 38 & 24 & 62 & & & & & & & \\
\cline { 2 - 10 } & Total & 44 & 31 & 75 & & & & & \\
\hline
\end{tabular}

$*, * *$ and $* * *$ stand for significance at 5,1 and $0.1 \%$ error margin, respectively

\section{Association of cocoa dieback disease with shade tree species}

Association between cocoa die back and presence of Maesopsis eminii was highly significant for disease incidence $(x 2=53.73), \quad(p<0.05)$; (Table 6). Association of the die back disease and presence of Cinnamomum tamala $(\mathrm{x} 2=8.07),(p<0.001)$; Persea Americana $(\mathrm{x} 2=14.10),(p<0.05) ;$ Eucalyptus globulus $(\mathrm{x} 2=25.01),(p<0.05) ;$ Schefflera actinophylla $(\mathrm{x} 2=4.24),(p<0.001) ;$ Mangifera indica $(\mathrm{x} 2=5.45),(p<0.05)$ showed high significance levels though these trees were planted on few farms. There was a non-significant association between die back with presence of Markhamia obtusifolia, Artocarpus heterophyllus, Triplochiton seleroxylon, Albizia glaberrima, Glicidia sepium, Arecaceae, Ficus capensis, Psidium guajava, Citrus X sinensis, Aleurites moluccanus, Spathodea campanulate, Morinda lucida.

Table 6: Cocoa Dieback Disease Prevalence with tree species

\begin{tabular}{|c|c|c|c|c|c|c|c|c|c|c|c|}
\hline \multirow{2}{*}{\multicolumn{2}{|c|}{ Shade tree species }} & \multicolumn{2}{|c|}{$\begin{array}{l}\text { Disease } \\
\text { presence }\end{array}$} & \multirow[t]{2}{*}{ Total } & \multirow[t]{2}{*}{$\begin{array}{l}\text { Chi- } \\
\text { squared }\end{array}$} & \multirow{2}{*}{\multicolumn{2}{|c|}{ Shade tree species }} & \multicolumn{2}{|c|}{$\begin{array}{l}\text { Disease } \\
\text { presence }\end{array}$} & \multirow[t]{2}{*}{ Total } & \multirow[t]{2}{*}{$\begin{array}{l}\text { Chi- } \\
\text { squared }\end{array}$} \\
\hline & & \multirow{2}{*}{\begin{tabular}{|l|} 
Yes \\
58
\end{tabular}} & \multirow{2}{*}{\begin{tabular}{|l|} 
No \\
0 \\
\end{tabular}} & & & & & \multirow{2}{*}{$\begin{array}{l}\text { Yes } \\
11\end{array}$} & \multirow{2}{*}{$\begin{array}{l}\text { No } \\
13\end{array}$} & & \\
\hline Maesopsis & Yes & & & 58 & $52.73 * * *$ & Eucalyptus & Yes & & & 24 & $25.01 * * *$ \\
\hline eminii & No & 6 & 16 & 22 & & globulus & No & 53 & 3 & 56 & \\
\hline & Total & 64 & 16 & 80 & & & Total & 64 & 16 & 80 & \\
\hline \multirow{3}{*}{$\begin{array}{l}\text { Markhamia } \\
\text { obtusifolia }\end{array}$} & Yes & 12 & 0 & 12 & \multirow[t]{3}{*}{3.53} & \multirow{3}{*}{$\begin{array}{l}\text { Pinus } \\
\text { sabiniana }\end{array}$} & Yes & 3 & 0 & 3 & \multirow[t]{3}{*}{0.78} \\
\hline & No & 52 & 16 & 68 & & & No & 61 & 16 & 77 & \\
\hline & Total & 64 & 16 & 80 & & & Total & 64 & 16 & 80 & \\
\hline \multirow{3}{*}{$\begin{array}{l}\text { Schefflera } \\
\text { actinophylla }\end{array}$} & Yes & 14 & 0 & 14 & \multirow[t]{3}{*}{$4.24 *$} & \multirow[t]{3}{*}{ Arecaceae } & Yes & 7 & 4 & 11 & \multirow[t]{3}{*}{2.13} \\
\hline & No & 50 & 16 & 66 & & & No & 57 & 12 & 69 & \\
\hline & Total & 64 & 16 & 80 & & & Total & 64 & 16 & 80 & \\
\hline \multirow{3}{*}{$\begin{array}{l}\text { Cinnamomum } \\
\text { tamala }\end{array}$} & Yes & 23 & 0 & 23 & \multirow[t]{3}{*}{$8.07 * * *$} & \multirow{3}{*}{$\begin{array}{l}\text { Ficus } \\
\text { capensis }\end{array}$} & Yes & 2 & 0 & 2 & \multirow[t]{3}{*}{0.51} \\
\hline & No & 41 & 16 & 57 & & & No & 62 & 16 & 78 & \\
\hline & Total & 64 & 16 & 80 & & & Total & 64 & 16 & 80 & \\
\hline \multirow{3}{*}{$\begin{array}{l}\text { Artocarpus } \\
\text { heterophyllus }\end{array}$} & Yes & 19 & 7 & 26 & \multirow[t]{3}{*}{1.15} & \multirow{3}{*}{$\begin{array}{l}\text { Psidium } \\
\text { guajava }\end{array}$} & Yes & 2 & 0 & 2 & 0.51 \\
\hline & No & 45 & 9 & 54 & & & No & 62 & 16 & 78 & \\
\hline & Total & 64 & 16 & 80 & & & Total & 64 & 16 & 80 & \\
\hline Triplochiton & Yes & 4 & 1 & 5 & 0 & Citrus & Yes & 1 & 1 & 2 & 5.28 \\
\hline seleroxylon & No & 60 & 15 & 75 & & sinensis & No & 63 & 14 & 77 & \\
\hline & Total & 64 & 16 & 80 & & & Total & 64 & 15 & 79 & \\
\hline Persea & Yes & 16 & 12 & 28 & $14.10 * * *$ & Aleurites & Yes & 10 & 0 & 10 & 2.86 \\
\hline Americana & No & 47 & 4 & 51 & & moluccanus & No & 54 & 16 & 70 & \\
\hline & Total & 63 & 16 & 79 & & & Total & 64 & 16 & 80 & \\
\hline Albizia & Yes & 1 & 0 & 1 & 0.25 & Spathodea & Yes & 1 & 0 & 1 & 0.25 \\
\hline glaberrima & No & 63 & 16 & 79 & & campanulata & No & 63 & 16 & 79 & \\
\hline & Total & 64 & 16 & 80 & & & Total & 64 & 16 & 80 & \\
\hline Glicidia & Yes & 5 & 0 & 5 & 1.33 & Morinda & Yes & 2 & 0 & 2 & 0.51 \\
\hline sepium & No & 59 & 16 & 75 & & lucida & No & 62 & 16 & 78 & \\
\hline & Total & 64 & 16 & 80 & & & Total & 64 & 16 & 80 & \\
\hline Mangifera & Yes & 8 & 6 & 14 & $5.54 *$ & & & & & & \\
\hline indica & No & 56 & 10 & 66 & & & & & & & \\
\hline & Total & 64 & 16 & 80 & & & & & & & \\
\hline
\end{tabular}

$*, * *$ and $* * *$ stand for significance at 5,1 and $0.1 \%$ error margin, respectively

\section{Discussion}

Influence of shade tree diversity on disease incidence 
The study considered eighty-two respondents with farms which are five acres and above from the four sub counties in Bundibugyo (Table 1).

Results focused on five cocoa diseases and their incidences in relation to the six shade tree diversities. The diseases were, black pod, witch's broom, frost pod rot, cocoa swollen shoot virus and cocoa die back. Cocoa plantations have always been associated with several diseases. (Gabriella Galarza Ferrin. August, 2019)

Shade diversity 1 (one tree species), shade diversity 2 (two tree species), shade diversity 3 (three tree species), shade diversity 4 (four tree species), shade diversity 5 (five tree species), and shade diversity 6 (six tree species) (Fig. 1-5). Cocoa diversity systems have special attention because of their economic, social, and ecological importance. (Hervé Bertin Daghela Bisseleua et al., 2014)

Association between diseases and presence of shade diversity varied depending on disease type as indicated in the results, the Parten of the association was not uniform in all diseases and this could have been influenced by several factors not limited to soil fertility, biodiversity issues, age of cocoa plantation, shade characteristics and the cocoa density. Agroforestry is a land use management system in which trees or shrubs are grown with crops and pasture land. It combines agricultural and forestry technologies to create more diverse, productive, profitable, healthy and sustainable land-use systems (James et al.,2014).

Shaded cocoa plantations, facilitate dispersal of forest fauna between fragments. Plants and animal biodiversity found within shaded coca systems could augment ecosystem series like pest control, pollination, weed control, fungal disease limitation, and soil fertility. (Hervé Bertin Daghela Bisseleua et al., 2014)

The association between pod rot disease and shade diversity presence was non-significant. Disease incidence was reported on the farms regardless of the type shade diversity present on farm.

Association between witch's broom disease incidence and presence of shade tree diversity was significant. This could have been because of cocoa age, most of the cocoa plantations were above 10 year of existence. The age of the cocoa plantation has a negative significant effect on the witches' broom disease control, low witch's broom incidence is associated with younger cocoa plantations. Spaggiari Souza et al. (2009) also found that there is an effect of cocoa age on the incidence of witch's broom disease during a 14- year trial in Brazil. It is argued that older and larger trees have more meristematic fluid which favor the inoculum production and dissemination of witch's broom. The result of this research agrees with the discussion because most of the farms were above 14 years of existence

Associations between Black pod, frost, swollen, cocoa dieback and presence of shade diversity was nonsignificant. The incidence of these diseases had no clear Parten of occurrence on selected farms for this research. Marius Wessel et al., (2015) says cocoa swollen virus and phytophthera pod rot are common diseases of cocoa, therefore they could have been caused by other factors. This calls for further studies on the specific causes of such diseases within the cocoa plantations but also identifying specific shade tree species of economic importance. Asare (2010) says the occurrence of trees on cocoa farms are as a result of natural regeneration and farmers do not have much to do with selection of specific tree species. During her analysis of shade establishment and management in cocoa farms in Ashanti region of Ghana showed that farmers primarily rely on natural regeneration to include shade trees on farms and this practice allows farmers to establish trees in a cost-effective manner that promotes species already in the land scape. However, these practices limit tree diversification options since it only allows trees already in the system to generate easily and thrive.

\section{Effect of shade tree species on disease incidence}

The association between black pod rot disease and presence of all tree species was non-significant. The disease incidence was not influenced by presence of the tree species. The association between witch's broom, swollen shoot virus, die back, frosty pod rot and presence of Maesopsis eminii was highly significant. Maesopsis eminii is likely to be a host for cocoa diseases and farmers need to care planting it for a shade tree. Witch's broom disease incidence was highly significant with presence of Maesopsis eminii $(x 2=55.41),(p<0.05)$; there was a significant association between witch's broom disease incidence with presence of Eucalyptus globulus (x2=16.71), ( $p<0.05)$; Persea Americana ( $22=9.79),(p<0.001)$; Markhamia 
obtusifolia ( $\mathrm{x} 2=3.95),(p<0.001) ;$ Mangifera indica $(\mathrm{X} 2=6.46),(p<0.001)$; and Schefflera actinophylla $(x 2=4.32),(p<0.001)$; however, these tree species were planted in limited numbers.

There was a significant association between Frosty rot disease incidence and presence of Maesopsis eminii with $(\mathrm{X} 2=25.90),(p<0.05)$. Association between frost incidence and Eucalyptus globulus $(\mathrm{X} 2=6.76)$, $(p<0.05)$; Mangifera indica $\left(\mathrm{X}^{2}=4.85\right),(p<0.001)$; is significant despite these tree species being planted in small numbers by farmers.

Association between swollen shoot virus with presence of Maesopsis eminii and Cinnamomum tamala was highly significant $\left(\mathrm{x}^{2}=31.55\right),(p<0.05) ;(\mathrm{x} 2=19.22),(p<0.05)$ was highly significant. Association between die back disease incidence with presence of maesopsis eminii was highly significant $(\mathrm{X} 2=52.73),(p<0.05)$. Marius Wessel et al., (2015) says cocoa swollen virus and phytophthera pod rot are common diseases of cocoa,

Trees are applied to crops for purposes of shade environment, reduce costs of weeding, control disease attack and increase the quality and taste of cocoa but should be selected trees. (Ngala, 2015). In other studies, some trees have been kept on farm for multipurpose uses in form of timber, fodder, fruit trees and biodiversity services. Abebe Mekoya Kassa, (2008).

Table 7. indicates a summary of disease incidences with different trees species on selected cocoa farms. There was a non-significant association between diseases under this study with presence of these tree species, Artocarpus heterophyllus, Triplochiton seleroxylon, Albizia glaberrima, Glicidia sepium, Pinus sabiniana, Arecaceae, Ficus capensis, Psidium guajava, Spathodea campanulate, Morinda, and farmers need to be encouraged to plant such trees for shade and agroecological purposes. Angelina Sanderson Bellamy, (2017) says practical agroecology is seen as a systemic and holistic approach for the farm's ecosystems. Core techniques and practices include: minimizing and ideally omitting chemical nutrients and high energy use; making use of the properties of the whole farming systems like recycling nutrients, building the soil organic matter, preserving Agro-biodiversity and resources.

Basing on the results, specific shade tree species need to be carefully identified and the technique of inter planting them on the cocoa plantations. These could be multipurpose trees which comprehensively boost the ecosystem services for the farmers' livelihoods. There is a gap on which tree species work better for cocoa shade in Bundibugyo district and this needs to be addressed. It was studied that the diversity of shade and the density of shade are important factors of the structure of cocoa agroforestry systems, which determine the provision of ecosystem services. Gabriela Galarza Ferrín. August, (2019). Table 8 has both botanical and English name of the tree species under study.

\section{Conclusion}

A fundamental feature of cocoa agroforestry systems is their variation in complexity. Shade structure complexity is especially important to be characterized. This research therefore, emphasizes the need for specific shade tree diversity to suit the purpose of the cocoa shade needs especially disease control, fertility improvement by recycling biomass, pest and disease management as well other Agro biodiversity services (James et al.,2014).

Specific shade tree species are very paramount in the cocoa plantations for composition of good shade diversity systems and provision of shade, disease control and others services. Care and thorough cautions should be taken in selecting which species to be considered for maximizing the output use of these tree species as opposed to Asare (2010) who says the occurrence of trees on cocoa farms are as a result of natural regeneration and farmers do not have much to do with selection of specific tree species. During her analysis of shade establishment and management in cocoa farms in Ashanti region of Ghana showed that farmers primarily rely on natural regeneration to include shade trees on farms and this practice allows farmers to establish trees in a cost-effective manner that promotes species already in the land scape.

This research identified most diseases being fungal, it therefore, encourages and recommends the farmers to use alternative measures to control these cocoa diseases and these should be ecofriendly measures but not 
limited to; planting cocoa disease tolerant varieties, pruning, planting non disease host trees, repellant trees, timely weeding, timely harvesting of ready cocoa pods, practicing agroforestry and mulching.

\section{Ethical considerations and approval}

Ethical aspects were highly observed through the process of this research. At university level, an authorization letter was issued allowing the study to proceed. High confidentiality was ensured during the administering of data collection tools. Questionnaires were administered at individual farmer level and their names were reserved not to be put on the questionnaire. Observations and photo graphs were taken after seeking authority from the owners of the specific fields in context.

\section{Acknowledgement}

This study was supported by African Centre of Excellence in Agroecology and Livelihood Systems (ACALISE) and the French Republic through Embassy of France to Uganda under an FSPI project 'Demonstration of a sustainable farm ecosystem for improved livelihoods of smallholder farmers in Uganda, http://odh.umu.ac.ug/'.

\section{References}

[1] (Amin, 2005). Adoption and effects of long-term supplementation on sheep performance. [publisher not identified], Wageningen.

[2] Amin. 2005. Foundations of statistical inference for social science research.

[3] Blandon.P. (1985). Agroforestry and portfolio theory. Agroforesyrt systems, 3(3).

[4] Colombia, G. and Nicaragua, P. Kenya, R. n.d. DOCUMENTED SPECIES DISTRIBUTION.

[5] Duguma, B. Gockowski, J. and Bakala, J. 2001. Smallholder cacao (Theobroma cacao Linn) cultivation in agroforestry systems of West and Central Africa:challenges and opportunities. Agroforestry Systems, 177-188.

[6] FAO. 2013. Agroforestry, food and nutritional security. International Conference on Forests for FoodSecurity and Nutrition. Rome, Italy.

[7] Huxley, P.A. and Westley, S.B. 1989. International Council for Research in Agroforestry (Eds.), 1989. Multipurpose trees: selection and testing for agroforestry: multipurpose trees and shrubs for specified agroforestry technologies and land-use systems--the ICRAF approach. International Council for Research in Agroforestry, Nairobi, Kenya.

[8] Kellimore, L.R. (Ed.), 2010. Handbook on agroforestry: management practices and environmental impact, Environmental science, engineering and technology series. Nova Science Publishers, New York.

[9] Mkandawire, P.T., Soludo, C.C. Mkandawire, P.T. (Eds.), 2003. African voices on structural adjustment: a companion to our continet, our future. International Development Research

[10] Centre; Africa World Press ; Council for the Development of Social Science Research in Africa, Ottawa : Trenton, N.J. : Dakar, Senegal.

[11] Mugenda, O. and Mugenda, A. 1991. Research Method Quantitative and qualitative Methods. Nairobi Kenya.

[12] NGALA, T.J. 2015a. Effect of shade trees on cocoa yield in small-holder cocoa (Theobroma cacao) Agroforests in Talba, Centre Cameroon (PhD Thesis). University of Dschang.

[13] NGALA, T.J. 2015b. Effect of shade trees on cocoa yield in small-holder cocoa (Theobroma cacao) agroforests in Talba, Centre Cameroon (PhD Thesis). University of Dschang.

[14] Owino, F. 1992. Improving multipurpose tree and shrub species for agroforestry systems. Agroforestry. Syst. 19, 131-137.

[15] Petithuguenin, P. 2000. The situation of cocoa production in Uganda First consultancy progress report for the.ADCIIDEA Project.

[16] Reeves, L.H. and Lilieholm, R.J. 1993. Reducing financial risk in agroforestry planning: a case study in Costa Rica. Agroforestry Systems, 169-175.

[17] Wessel, M. and Quist-Wessel, P.M.F. 2015.Cocoa production in West Africa, a review and analysis of recent developments. NJAS - Wagening. J. Life Sci. 74-75, 1-7. https://doi.org/10.1016/j.njas.2015.09.001 


\section{Appendices1.}

Table 7: Disease Association with specific shade tree species

\begin{tabular}{|l|l|l|l|l|l|l|l|l|l|}
\hline Tree species & Witch's & Frost Pd & Swollen & Die back & Tree species & Witches & $\begin{array}{l}\text { Frost } \\
\text { Pd }\end{array}$ & Swollen & Die back \\
\hline $\begin{array}{l}\text { Maesopsis } \\
\text { eminii }\end{array}$ & $55.41^{* * *}$ & $25.90^{* * *}$ & $31.55^{* * *}$ & $52.73^{* * *}$ & $\begin{array}{l}\text { Eucalyptus } \\
\text { globulus }\end{array}$ & $16.71^{* * *}$ & $6.76^{* * *}$ & $14.52^{* * *}$ & $25.01^{* * *}$ \\
\hline $\begin{array}{l}\text { Markhamia } \\
\text { Obtusifolia }\end{array}$ & $3.95^{* *}$ & 2.04 & 0.38 & 3.53 & $\begin{array}{l}\text { Pinus } \\
\text { sabiniana }\end{array}$ & 0.79 & 0.41 & 0.08 & 0.78 \\
\hline $\begin{array}{l}\text { Schefflera } \\
\text { Actinophylla }\end{array}$ & $4.32^{*}$ & 2.23 & 1.7 & $4.24^{*}$ & Arecaceae & 2.76 & 0.08 & 2.62 & 2.13 \\
\hline $\begin{array}{l}\text { Cinnamomum } \\
\text { tamala }\end{array}$ & & & $19.22^{* * *}$ & $8.07^{* * *}$ & $\begin{array}{l}\text { Ficus } \\
\text { capensis }\end{array}$ & 0.53 & 0.27 & 0.06 & 0.51 \\
\hline $\begin{array}{l}\text { Artocarpus } \\
\text { heterophyllus }\end{array}$ & 2.65 & 0.01 & 1.93 & 1.15 & $\begin{array}{l}\text { Psidium } \\
\text { guajava }\end{array}$ & 0.52 & 0.27 & 0.06 & 0.51 \\
\hline $\begin{array}{l}\text { Triplochiton } \\
\text { seleroxylon }\end{array}$ & 0 & 0.71 & 0.8 & 0 & $\begin{array}{l}\text { Citrus } X \\
\text { sinensis }\end{array}$ & 5.19 & 3.09 & 1.51 & 5.28 \\
\hline $\begin{array}{l}\text { Persea } \\
\text { Americana }\end{array}$ & $9.79^{* * *}$ & 3.83 & $11.47^{* * *}$ & $14.10^{* * *}$ & $\begin{array}{l}\text { Aleurites } \\
\text { moluccanus }\end{array}$ & 2.58 & 1.32 & $7.21^{* * *}$ & 2.86 \\
\hline $\begin{array}{l}\text { Albizia } \\
\text { glaberrima }\end{array}$ & 0.26 & 1.13 & 1.44 & 0.25 & $\begin{array}{l}\text { Spathodea } \\
\text { campanulate }\end{array}$ & 0.26 & 0.31 & 0.71 & 0.23 \\
\hline $\begin{array}{l}\text { Glicidia } \\
\text { sepium } \\
\text { Mandica }\end{array}$ & 1.36 & 0.55 & 2.2 & 1.33 & $\begin{array}{l}\text { Morinda } \\
\text { lucida }\end{array}$ & 0.52 & 0.27 & 0.45 & 0.51 \\
\hline
\end{tabular}

\section{Appendices}

Table 8: Tree Species as recorded in the 82 farms of Bundibugyo District, western Uganda

\begin{tabular}{|l|l|l|l|}
\hline Species name & English name & Species name & English name \\
\hline Maesopsis eminii & Maesopsis & Eucalyptus globulus & Eucalyptus \\
\hline Markhamia obtusifolia & Markhamia & Pinus sabiniana & Pine \\
\hline Schefflera actinophylla & Umbrella & Arecaceae & Palm \\
\hline Artocarpus heterophyllus & Jack fruit & Ficus capensis & Ficus \\
\hline Triplochiton seleroxylon & $\begin{array}{l}\text { African white } \\
\text { wood }\end{array}$ & Citrus X sinensis & Guava \\
\hline Persea Americana & Avocado & Aleurites moluccanus & Candle nut \\
\hline Albizia glaberrima & Lowveld & Spathodea & African Tulip \\
\hline
\end{tabular}




\begin{tabular}{|l|l|l|l|}
\hline & & campanulate & \\
\hline Glicidia sepium & Gliricidia & Morinda lucida & Brimstone tree \\
\hline Mangifera indica & Mango & & \\
\hline
\end{tabular}

\section{Appendices 3}

Disease Photographs

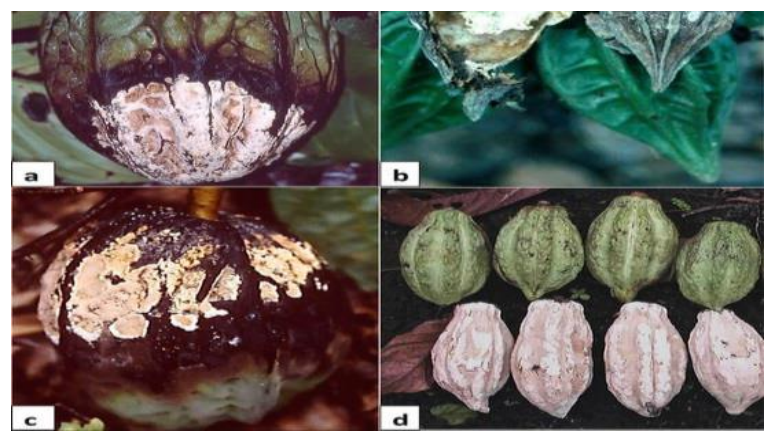

Frosty pod rot

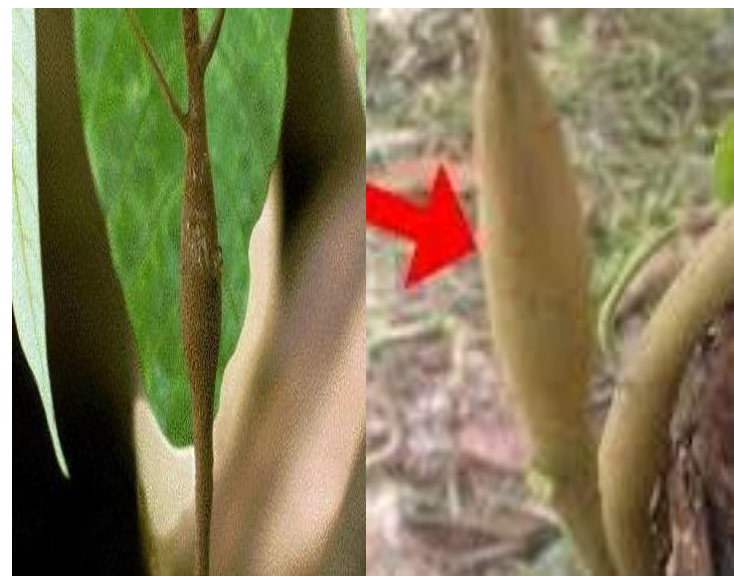

Cocoa swollen shoot virus

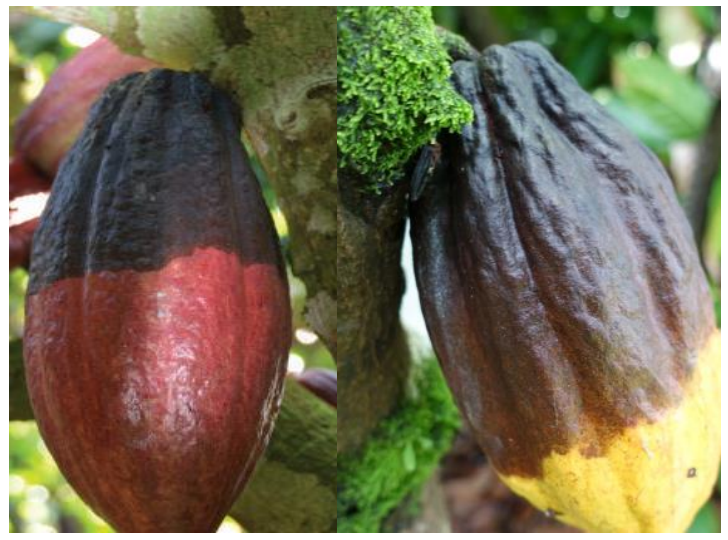

Black pod rot disease

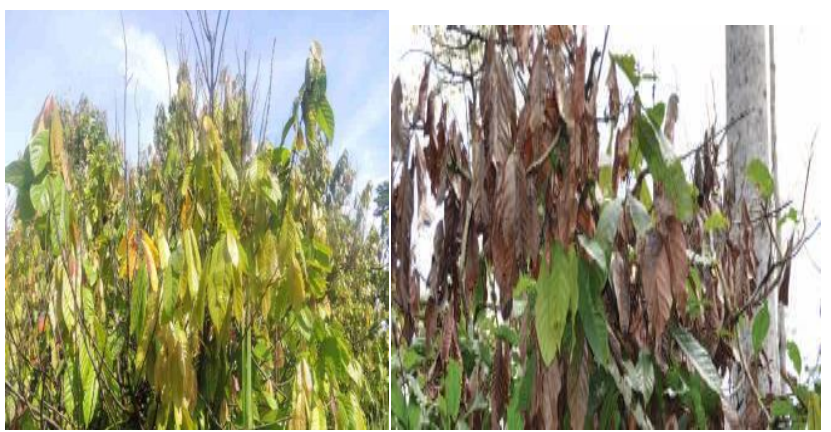

Cocoa dieback

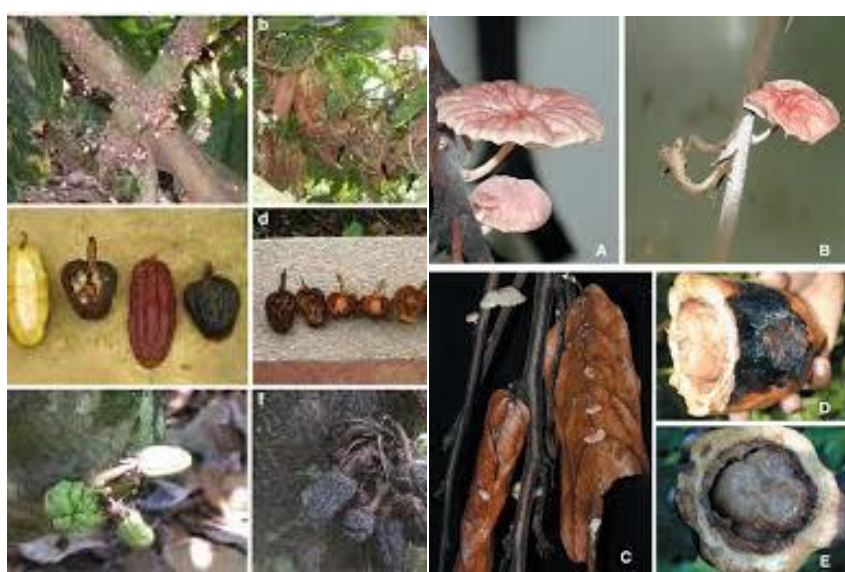

Witch's broom 